\title{
Indoor dust acts as an adjuvant to promote sensitization to peanut through the airway
}

\author{
Johanna M. Smeekens ${ }^{1,2}$ (D) | Robert M. Immormino ${ }^{1}$ | Peter A. Balogh ${ }^{1}$ | \\ Scott H. Randell ${ }^{3}$ | Michael D. Kulis ${ }^{1,2}$ (D) | Timothy P. Moran ${ }^{1}$
}

${ }^{1}$ Department of Pediatrics, UNC School of Medicine, Chapel Hill, NC

${ }^{2}$ UNC Food Allergy Initiative, Chapel Hill, NC

${ }^{3}$ Department of Cell Biology and

Physiology, UNC School of Medicine, Chapel Hill, NC

\footnotetext{
Correspondence

Johanna M. Smeekens, PhD, University of North Carolina at Chapel Hill, 116 Manning Dr. Mary Ellen Jones Building Room 3310, Chapel Hill, NC 27599 USA.

Email: smeeken3@email.unc.edu

Funding information

JMS is funded by a T32 Allergy/Immunology Training Grant (Al007062) through Duke University and University of North Carolina (UNC) at Chapel Hill. This work is supported in part by the American Research Foundation for Nut Allergies (MDK. and JMS), the American Academy of Allergy, Asthma and Immunology Foundation (TPM), the UNC Center for Environmental Health and Susceptibility (TPM and MDK.), the Cystic Fibrosis Foundation (BOUCHE15RO to SHR), and the NIH (DK065988 to SHR).
}

\begin{abstract}
Background: There is growing evidence that environmental peanut exposure through non-oral routes, including the skin and respiratory tract, can result in peanut sensitization. Environmental adjuvants in indoor dust can promote sensitization to inhaled antigens, but whether they contribute to peanut allergy development is unclear.

Objective: We investigated whether indoor dust promotes airway sensitization to peanut and peanut allergy development in mice.

Methods: Female and male C57BL/6J mice were exposed via the airways to peanut, indoor dust extract, or both for 2 weeks. Mice were then challenged with peanut and assessed for anaphylaxis. Peanut-specific immunoglobulins, peanut uptake by lung conventional dendritic cells (cDCs), lung innate cytokines, and T cell differentiation in lung-draining lymph nodes were quantified. Innate cytokine production by primary human bronchial epithelial cells exposed to indoor dust was also determined.
\end{abstract}

Results: Inhalational exposure to low levels of peanut in combination with indoor dust, but neither alone, resulted in production of peanut-specific IgE and development of anaphylaxis upon peanut challenge. Indoor dust triggered production of innate cytokines in murine lungs and in primary human bronchial epithelial cells. Additionally, inhaled indoor dust stimulated maturation and migration of peanut-laden lung type $1 \mathrm{cDCs}$ to draining lymph nodes. Inhalational exposure to peanut and indoor dust induced peanut-specific $T$ helper 2 cell differentiation and accumulation of $T$ follicular helper cells in draining lymph nodes, which were associated with increased B cell numbers and peanut-specific immunoglobulin production.

Conclusions \& clinical relevance: Indoor dust promotes airway sensitization to peanut and development of peanut allergy in mice. Our findings suggest that environmental adjuvants in indoor dust may be determinants of peanut allergy development in children.

KEYWORDS

airway sensitization, anaphylaxis, dendritic cells, indoor dust, peanut allergy, $\mathrm{T}$ follicular helper cells 


\section{1 | INTRODUCTION}

Peanut allergy (PA) is a growing public health concern, affecting roughly $2 \%$ of the population in industrialized countries. ${ }^{1,2} \mathrm{PA}$ is usually lifelong ${ }^{3}$ and is responsible for the majority of deaths related to food allergy. ${ }^{4}$ While early-life peanut consumption has shown promise in reducing PA development, ${ }^{5}$ a significant number of infants are already sensitized to peanut prior to introduction. ${ }^{5,6}$ How peanut sensitization develops during early infancy is unclear, but there is growing evidence that environmental peanut exposure plays an important role. ${ }^{7-10}$ Biologically active peanut is detectable in dust collected from homes, 9,11,12 and the levels of peanut allergen in indoor dust directly correlate with rates of peanut sensitization and probable PA in children at high risk for PA. ${ }^{8,9,13}$ However, environmental peanut exposure was not associated with peanut sensitization in children without atopic risk factors, ${ }^{13}$ suggesting that other indoor environmental factors may influence the risk of peanut sensitization. Understanding the environmental determinants of peanut sensitization will be essential for creating effective interventions aimed at preventing PA development.

The vast majority of PA subjects react upon their first known ingestion of peanut, ${ }^{14}$ suggesting sensitization occurs through nonoral routes of exposure. While there is evidence that cutaneous exposure to peanut through an impaired skin barrier can result in sensitization, ${ }^{8-10}$ recent studies in rodents have shown that inhalational exposure to peanut can also lead to sensitization and anaphylaxis to peanut allergen. ${ }^{15-17}$ In addition, peanut-specific $C D 4^{+} \mathrm{T}$ cells from PA subjects express both airway- and skin-homing chemokine receptors, suggesting that peanut sensitization may occur through the skin and respiratory tract. ${ }^{18}$ While peanut allergen is not thought to be airborne in homes, ${ }^{11}$ the physical proximity of infants to floors, as well as their rapid respiratory rates, likely increases their risk for inhalational exposure to peanut in indoor dust. ${ }^{19}$ Thus, inhalational exposure to environmental peanut is a plausible route for peanut sensitization during infancy.

Sensitization to inhaled allergens involves both innate and adaptive immune responses in the lungs. ${ }^{20}$ Inhaled allergens are taken up by lung conventional dendritic cells ( $c D C s)$, which then migrate to draining lymph nodes and present antigen to $\mathrm{CD} 4^{+} \mathrm{T}$ helper cells. ${ }^{21}$ Allergens also trigger airway epithelial cells to release innate cytokines, including interleukin (IL)-1, IL-33, and thymic stromal lymphopoietin (TSLP), which in turn programme lung CDCs to induce differentiation of allergen-specific T helper 2 (Th2) cells. ${ }^{22-24}$ Through the secretion of IL- 4 and IL-13, Th2 cells promote allergenspecific IgE production by B cells. ${ }^{25}$ Although Th2 cells have historically been considered the primary mediators of allergic sensitization, there is growing evidence that $\mathrm{T}$ follicular helper ( $\mathrm{Tfh}$ ) cells also play a critical role in promoting IgE production. ${ }^{15,26-28}$ Through their ability to provide help to germinal centre B cells, Tfh cells promote antibody isotype class switching and B cell differentiation into plasma and memory cells. ${ }^{29}$ Accordingly, recent studies have shown that Tfh cells are essential for the development of IgE responses against inhaled antigens. ${ }^{15,27,28}$ Like other $\mathrm{T}$ helper cell subsets, cDCs are necessary for priming antigen-specific Tfh cells. ${ }^{30-32}$ While several studies have investigated the role of lung $\mathrm{CDCs}$ in promoting Th2 responses, ${ }^{33-35}$ the mechanisms by which lung cDCs prime allergenspecific Tfh cells remain poorly understood.

In addition to food allergens, indoor dust contains a mixture of environmental agents with immunostimulatory properties, including endotoxin, fungal-derived polysaccharides, and proteases. ${ }^{36-39}$ These environmental agents can act as adjuvants in the respiratory tract and promote allergic sensitization to normally innocuous antigens in animal models. ${ }^{40-44}$ It is likely that the composition of environmental adjuvants in indoor dust can significantly influence sensitization and allergy development. Indeed, the bioactivity of indoor dust has been associated with aeroallergen sensitization in children at risk for allergy. ${ }^{37}$ We have previously shown that indoor dust extracts can programme lung cDCs to induce Th2 responses to inhaled antigens, resulting in the development of allergic airway inflammation upon subsequent inhalational allergen challenge. ${ }^{42,45}$ Whether adjuvants in indoor dust can also promote the development of allergy to environmental food allergens is unknown. To address this question, we developed an animal model that mimics inhalational exposure to environmental peanut allergen. We found that inhalational coexposure to peanut and indoor dust, but neither alone, induced peanut-specific IgE and IgG1 production. Importantly, mice sensitized with peanut and indoor dust developed anaphylaxis upon peanut challenge. Inhalational exposure to indoor dust triggered the release of innate cytokines in both murine lungs and primary human bronchial epithelial cells. Furthermore, inhaled indoor dust stimulated the maturation and migration of peanut-laden CDCs to lungdraining lymph nodes (LNs), which was associated with induction of peanut-specific Th2 and Tfh cell differentiation. Collectively, these findings suggest that environmental adjuvants in indoor dust are determinants for PA development in children.

\section{2 | METHODS}

\section{1 | Mice}

C57BL/6J were obtained from the Jackson Laboratories (Bar Harbor, ME) and bred at the University of North Carolina at Chapel Hill. All mice were housed in specific pathogen-free conditions, raised on standard mouse chow free of any peanut-ingredients, and kept on a 12:12-hour light/dark cycle. Female and male mice were used for experiments between 6 and 12 weeks of age. All animal experiments were approved by the Institutional Animal Care and Use Committee at the University of North Carolina at Chapel Hill.

\section{2 | Reagents}

Peanut allergen extract was prepared from roasted de-fatted peanut flour (Golden Peanut) in PBS with $1 \mathrm{M} \mathrm{NaCl}$ as described previously. ${ }^{46}$ Extracts of indoor dust (ID) were prepared as previously described. ${ }^{47}$ Briefly, vacuumed dust samples were collected from a single-family residence over several months to minimize sampling 
variability. Pooled dust samples were passed through a coarse sieve, weighed, and then extracted at $100 \mathrm{mg} / \mathrm{mL}$ with PBS at $4^{\circ} \mathrm{C}$ with mild agitation for 16 hours. The samples were centrifuged to remove insoluble debris, and supernatants were sterilized by passage through a $0.22-\mu \mathrm{m}$ filter. Endotoxin levels in the experimental ID samples were $73 \mathrm{ng} / \mathrm{mL}$ (730 EU/mL) as determined by Limulus amebocyte lysate assay (Thermo Scientific). Total peanut protein in ID samples was determined to be $172 \mathrm{ng} / \mathrm{mL}$ (or $1.72 \mu \mathrm{g} / \mathrm{g}$ dust) as measured by the Veratox for Peanut Allergen Assay (Neogen, Lansing, MI). Ambient levels of the major peanut allergens Ara $h$ 2 and 3 in ID samples were 3.6 and $8.4 \mathrm{ng} / \mathrm{mL}$, respectively, as measured by ELISA (Indoor Biotechnologies). Indoor dust extracts were aliquoted, stored at $-80^{\circ} \mathrm{C}$, and discarded after more than two freeze-thaw cycles.

\section{3 | Peanut airway sensitization and challenge model}

Mice were lightly anaesthetized by isoflurane inhalation and given 0.005-50 $\mu$ g of peanut alone or with $10 \mu \mathrm{L}$ of ID in a total volume of $50 \mu \mathrm{L}$ by oropharyngeal (o.p.) aspiration twice weekly for two weeks as previously described. ${ }^{41,48}$ Serum was collected by submandibular bleed at the indicated time-points for immunoglobulin quantification. Seven days after the last sensitization (day 17), mice were challenged to peanut ( $8 \mathrm{mg}$ protein) by intraperitoneal (i.p.) injection. Core body temperatures were monitored every 15 minutes with a rectal thermometer (Physitemp). Anaphylaxis was defined as a greater than $3^{\circ} \mathrm{C}$ decrease in body temperature.

\section{4 | ELISA for peanut-specific antibodies and cytokines}

Peanut-specific IgE, IgG1, and IgG2c were quantified via ELISA as described previously. ${ }^{49}$ Briefly, 96-well plates were coated with $20 \mu \mathrm{g} / \mathrm{mL}$ HSA-DNP (for standard curves) or peanut extract (for samples), and blocked with $2 \%$ BSA in PBS-0.5\% Tween. Samples were diluted 1:20 for peanut-specific IgE, 1:20 000 for peanut-specific IgG1, and 1:10 000 for peanut-specific IgG2c ELISAs. Standard curves ranging from 0.002 to $2 \mu \mathrm{g} / \mathrm{mL}$ of either mouse IgE anti-DNP, IgG1 anti-DNP, or IgG2c anti-DNP (Accurate Chemicals) were generated via 1:2 serial dilutions. IgE plates were detected with the following antibodies in succession: sheep IgG anti-mouse IgE $(0.5 \mu \mathrm{g} /$ $\mathrm{mL}$, The Binding Site), biotinylated donkey anti-sheep IgG $(0.5 \mu \mathrm{g} /$ $\mathrm{mL}$, Accurate Chemicals), and NeutrAvidin-HRP $(0.5 \mu \mathrm{g} / \mathrm{mL}$, Pierce Biotechnology). IgG1 and IgG2c plates were detected with HRP-goat anti-mouse IgG1 (1:40 000, Southern Biotech) or IgG2c (1:20 000, Southern Biotech), respectively. All plates were developed using TMB (SeraCare) and stopped using $1 \% \mathrm{HCl}$ (SeraCare). Immunoglobulin ELISA plates were read at $450 \mathrm{~nm}$ using a microplate spectrophotometer (BioTek Instruments). Measurement of mouse cytokines was performed using the following commercial ELISA kits: IL- $1 \alpha$, IL-1 $\beta$, and IL-33 (R\&D Systems); IL-4, TSLP, and IFN- $\gamma$ (BioLegend); IL-13 (eBioscience); and IL-5 (BD Biosciences).

\section{5 | Cytokine production by lung-draining LNs}

Mice were exposed to peanut, ID, or both via the airways twice weekly for 2 weeks as described above. On day 14, mediastinal LNs (mLNs) were harvested and passed through a $70-\mu \mathrm{m}$ strainer to obtain a single-cell suspension. LN cells ( 200000 cells/well) were cultured for 4 days in complete RPMI 10 medium (RPMI-1640, 10\% fetal bovine serum (Gemini), penicillin-streptomycin, $50 \mu \mathrm{mol} / \mathrm{L} \mathrm{2-}$ mercaptoethanol, $10 \mathrm{mmol} / \mathrm{L} \mathrm{HEPES})$ and peanut $(100 \mu \mathrm{g} / \mathrm{mL})$ in a 96-well round-bottom plate. Supernatants were collected 4 days later, and cytokines were measured by ELISA.

\subsection{Analysis of cytokines in whole lungs}

Mice were exposed to peanut, ID, or both via the airways by o.p. administration. Six hours later, lungs were collected, weighed, and snap-frozen in liquid nitrogen. Frozen lungs were suspended in PBS with protease inhibitors (Roche Diagnostics) at 1.5 volumes (g lung/mL PBS) and homogenized using a VWR-200 homogenizer. Total protein content was determined with a BCA assay (Pierce Biotechnology). Cytokine levels were measured by ELISA and normalized to total protein in the lung homogenates.

\section{7 | Primary human bronchial epithelial cell (HBEC) culture and cytokine analysis}

Human donor lungs unsuitable for transplantation were obtained following informed consent for research use under protocol \#03-1396, approved by the University of North Carolina at Chapel Hill Biomedical Institutional Review Board. Primary human bronchial epithelial cells (HBECs) were obtained by enzymatic digestion as described. ${ }^{50}$ Cells from three non-smoker donors with no prior history of chronic lung disease were grown on plastic in bronchial epithelial growth media for two passages before culture under air-liquid interface (ALI) conditions on culture inserts (6.5 mm diameter, polyester $0.4 \mu \mathrm{m}$ pore size; Costar 3470, Fisher Scientific). Peanut $(50 \mu \mathrm{g} / \mathrm{mL})$, ID extract $(10 \% \mathrm{v} / \mathrm{v})$, or both were applied to the apical cell surface of fully differentiated HBEC cultures. Four hours later, apical washes were collected for cytokine analysis. Measurement of human cytokines was performed using IL-1 $\alpha$, IL-1 $\beta$, IL-33, and TSLP ELISA kits (R\&D Systems).

\subsection{Flow cytometric analysis of lung and LN cells}

Murine lung and LN cells were isolated and analysed by flow cytometry as previously described. ${ }^{51,52}$ Briefly, harvested lungs and $\mathrm{mLNs}$ were manually cut with scissors and digested with Liberase

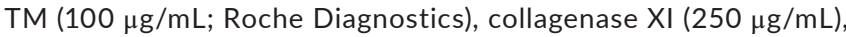
hyaluronidase 1a (1 mg/mL), and DNase I (200 $\mu \mathrm{g} / \mathrm{mL}$; Sigma, St. Louis, MO) for 1 hour (lungs) or 30 minutes ( $\mathrm{mLNs}$ ) at $37^{\circ} \mathrm{C}$. The digested tissue was passed through a 70- $\mu \mathrm{m}$ nylon strainer to obtain a single-cell suspension. Red blood cells were lysed with $0.15 \mathrm{M}$ ammonium chloride and $1 \mathrm{mmol} / \mathrm{L}$ potassium bicarbonate 
(ACK lysis). Cells were incubated with anti-mouse CD16/CD32 (2.4G2) for 5 minutes to block Fc receptors, followed by incubation with fluorochrome- or biotin-conjugated antibodies against murine $\mathrm{CD} 3 \varepsilon$ (145-2C11), CD4 (GK1.5), CD11b (M1/70), CD11c (N418), CD19 (6D5), CD44 (IM7), CD45 (30-F11), CD88 (20/70), CD103 (M290), Ly-6C (AL-21), Ly-6G (1A8), I-A/E (M5/114.15.2), CXCR5 (L138D7), PD-1 (29F.1A12), and Siglec-F (E50-2440) for 30 minutes on ice. Staining with biotinylated antibodies was followed by incubation with fluorochrome-conjugated streptavidin for 20 minutes on ice. Cells were also concurrently stained with Zombie Aqua (BioLegend) for live cell/dead cell discrimination. Flow cytometry data were acquired with a four-laser LSRII (BD Biosciences) and analysed using FlowJo (Ashland, OR) software. Only single cells were analysed. All antibodies were purchased from BioLegend or BD Biosciences. In some experiments, peanut uptake by lung CDCs was evaluated following instillation of peanut labelled with Alexa Fluor 647 (Invitrogen) as described previously. ${ }^{51,52}$

\section{9 | Statistical analysis}

GraphPad Prism version 8 was used to analyse all data. Paired $t$ tests, and one- or two-way ANOVA tests were performed, and a $P$ value $<.05$ was considered significant.

\section{3 | RESULTS}

\section{1 | Environmental adjuvants in indoor dust promote airway sensitization to peanut}

Prior studies have shown that inhalational exposure to peanut and other food allergens can result in sensitization. ${ }^{15-17}$ However, these studies involved airway delivery of either allergen alone or in combination with cholera toxin as an adjuvant. To more accurately replicate natural exposure to environmental peanut in within the home, we coadministered peanut $(50 \mu \mathrm{g})$ with indoor dust extract (ID) to the airways of mice twice weekly for two weeks (Figure 1A). Inhalational exposure to peanut and ID resulted in marked production of peanut-specific IgE, IgG1, and IgG2c (Figure 1B). Peanut-specific IgE and IgG1 were detectable as early as one week after the initial exposure, whereas IgG2c was not detectable until after two weeks of exposure (Figure 1B). In contrast, inhalational exposure to peanut alone induced only modest levels of peanut-specific IgE and very low levels of IgG1. Consistent with the very low levels of peanut allergen measured in our ID preparation, inhalational exposure to ID alone did not induce peanut-specific immunoglobulins. Thus, ID has potent adjuvant activity that promotes airway sensitization to inhaled peanut.
FIGURE 1 Indoor dust promotes airway sensitization to peanut. A, Experimental schematic for peanut sensitization. B, Peanut-specific IgE, $\operatorname{lgG1}$, and IgG2c quantified on days 0,7 , and 14 of sensitization regimen. $\mathrm{C}$, Core body temperatures recorded after i.p. challenge to peanut. Symbols represent means \pm SEM ( $n=6$ mice per group). Data shown are from a single experiment, representative of three experiments. Statistical comparisons are between $\mathrm{PN}+\mathrm{ID}$ and PN groups. ${ }^{*} \mathrm{P}<.05,{ }^{* *} \mathrm{P}<.01$, ${ }^{* * *} P<.001$, two-way ANOVA. ID, indoor dust; PN, peanut
(A) $\quad$ C57BL/6J (age 6-10 wk)



Day:

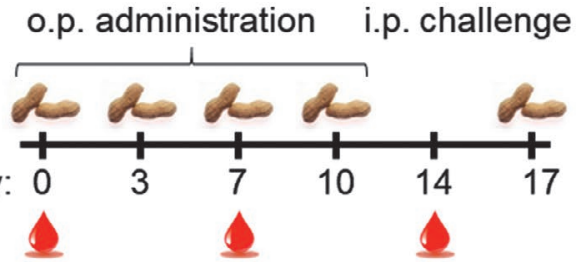

(B)

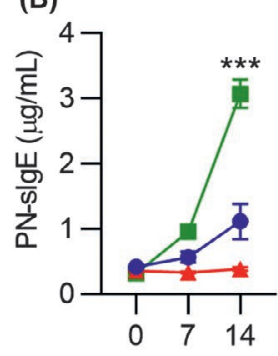

Time (days)
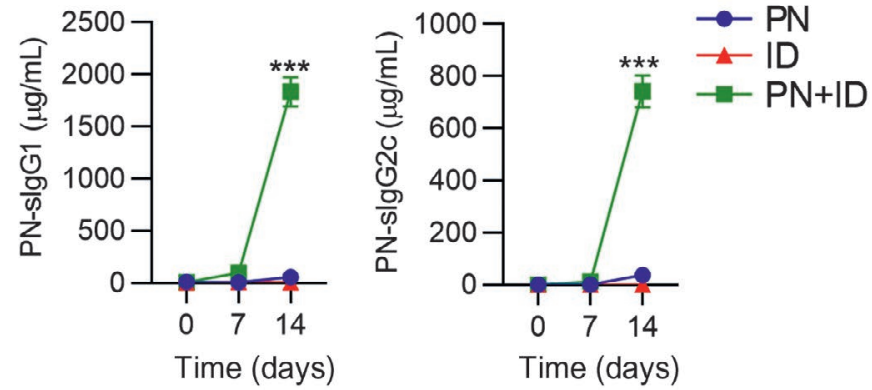

(C)




To determine whether airway sensitization to peanut would result in clinical reactivity upon systemic challenge, sensitized mice were administered an i.p. injection of peanut allergen. Mice sensitized with peanut and ID exhibited a dramatic decrease in body temperature after challenge, consistent with the development of anaphylaxis (Figure 1C). In contrast, mice sensitized with neither ID nor peanut alone exhibited a substantial decrease in body temperature after challenge (Figure $1 \mathrm{C}$ ). Taken together, these findings demonstrate that environmental adjuvants in ID promote sensitization to inhaled peanut, resulting in the development of anaphylaxis after systemic challenge.

\subsection{Indoor dust induces sensitization to low levels of inhaled peanut}

In the United States, environmental sampling studies have reported the median peanut concentration in household dust to be roughly $25-40 \mu \mathrm{g} / \mathrm{g} .{ }^{9,53}$ It is estimated that infants are exposed to $50-100 \mathrm{mg}$ of indoor dust daily, ${ }^{54}$ indicating that average environmental exposure to peanut allergen is likely low. We therefore investigated whether ID would promote sensitization to low levels of peanut allergen. When combined with ID, inhalational exposure to as little as $500 \mathrm{ng}$ of peanut resulted in sensitization, as evident by production of peanut-specific IgE and IgG1 (Figure 2A). Importantly, mice exposed to 500 ng of peanut in combination with ID demonstrated anaphylaxis upon peanut challenge (Figure 2B). Exposure to $5 \mathrm{ng}$ of peanut and ID did not result in sensitization or anaphylaxis upon peanut challenge (Figure $2 A, B$ ), indicating that the threshold for peanut sensitization in our model was in the range of 5-500 ng of peanut allergen. Furthermore, exposure to ID alone, which contained $1.72 \mathrm{ng}$ peanut protein per o.p. administration, nor peanut alone, resulted in sensitization or anaphylaxis upon peanut challenge (Figure 2A,B). Thus, adjuvants in ID facilitate sensitization to low and likely environmentally relevant levels of peanut allergen.

\section{3 | Inhaled indoor dust induces innate cytokine production in murine lungs and primary HBECs}

Stimulation of innate immune responses is a critical step for allergic sensitization in the lungs. ${ }^{25,55}$ Inhaled allergens and other noxious agents can trigger release of innate cytokines (IL-33, TSLP, IL-1) by airway epithelial cells, which in turn act upon DCs and innate lymphoid cells to promote Th2 responses. ${ }^{20}$ Furthermore, IL-1 signalling has also been shown to promote allergen-specific IgE production to inhaled allergens. ${ }^{15}$ We therefore investigated whether environmental adjuvants in ID stimulated innate cytokine production in the lungs. Inhalational exposure to ID resulted in a marked increase in IL-1 $\alpha, \mathrm{IL}-1 \beta$, and IL-33 in the lungs at 6 hours post-exposure (Figure 3A). Consistent with prior studies, ${ }^{15}$ inhalational exposure to peanut alone induced a modest increase in lung levels of IL- $1 \alpha$ and IL-1 $\beta$, but not IL-33 (Figure 3A). Inhalational exposure to combined peanut and ID induced similar levels of IL-1 $\alpha$ and IL-1 $\beta$ cytokines compared to ID alone, indicating that ID and peanut did not have synergistic effects on innate cytokine production (Figure 3A). Interestingly, TSLP lung levels were increased in mice exposed to peanut but not ID, suggesting that peanut can directly stimulate TSLP production (Figure 3A).

We next investigated whether ID could induce innate cytokine production by human airway epithelial cells. Treatment of primary HBECs with ID for four hours resulted in apical secretion of IL- $1 \alpha$ and IL-1 $\beta$ (Figure 3B). Treatment of HBECs with peanut alone did not induce innate cytokine production (Figure 3B), suggesting that the endogenous adjuvant activity of peanut is not due to direct stimulation of airway epithelial cells. We did not detect induction of IL-33 or TSLP in peanut- or ID-treated HBECs (data not shown), although we cannot exclude the possibility that these cytokines are produced at later time-points. Taken together, these findings indicate that acute exposure to ID can induce innate cytokine production in murine lungs and in human airway epithelial cells.

(A)



(B)

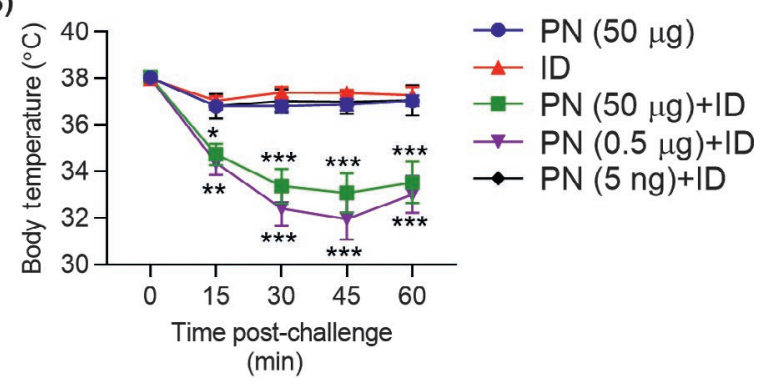

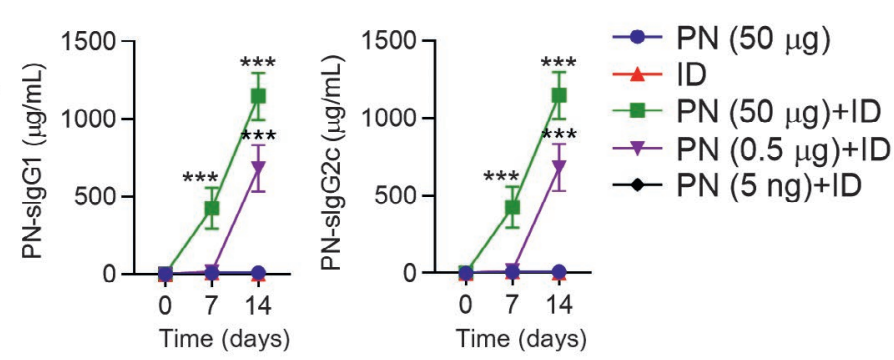

$\rightarrow$ PN $(50 \mu g)$

- ID

- PN $(50 \mu \mathrm{g})+\mathrm{ID}$

$\sim \operatorname{PN}(0.5 \mu \mathrm{g})+\mathrm{ID}$
FIGURE 2 Indoor dust induces sensitization to low levels of inhaled peanut. A, Peanut-specific IgE, IgG1, and IgG2c quantified on days 0,7 and 14 of sensitization regimen. Mice were sensitized with varying amounts of PN (50 $\mu \mathrm{g}, 0.5 \mu \mathrm{g}, 5 \mathrm{ng})+$ ID. B, Core body temperatures recorded after i.p. challenge to peanut. Symbols represent means \pm SEM of pooled data from 3 experiments ( $n=5-12$ mice per group). Statistical comparisons are between $\mathrm{PN}+\mathrm{ID}$ and PN groups. ${ }^{*} \mathrm{P}<.05,{ }^{* *} \mathrm{P}<.01$, ${ }^{* * *} P<.001$, two-way ANOVA. ID, indoor dust; PN, peanut 

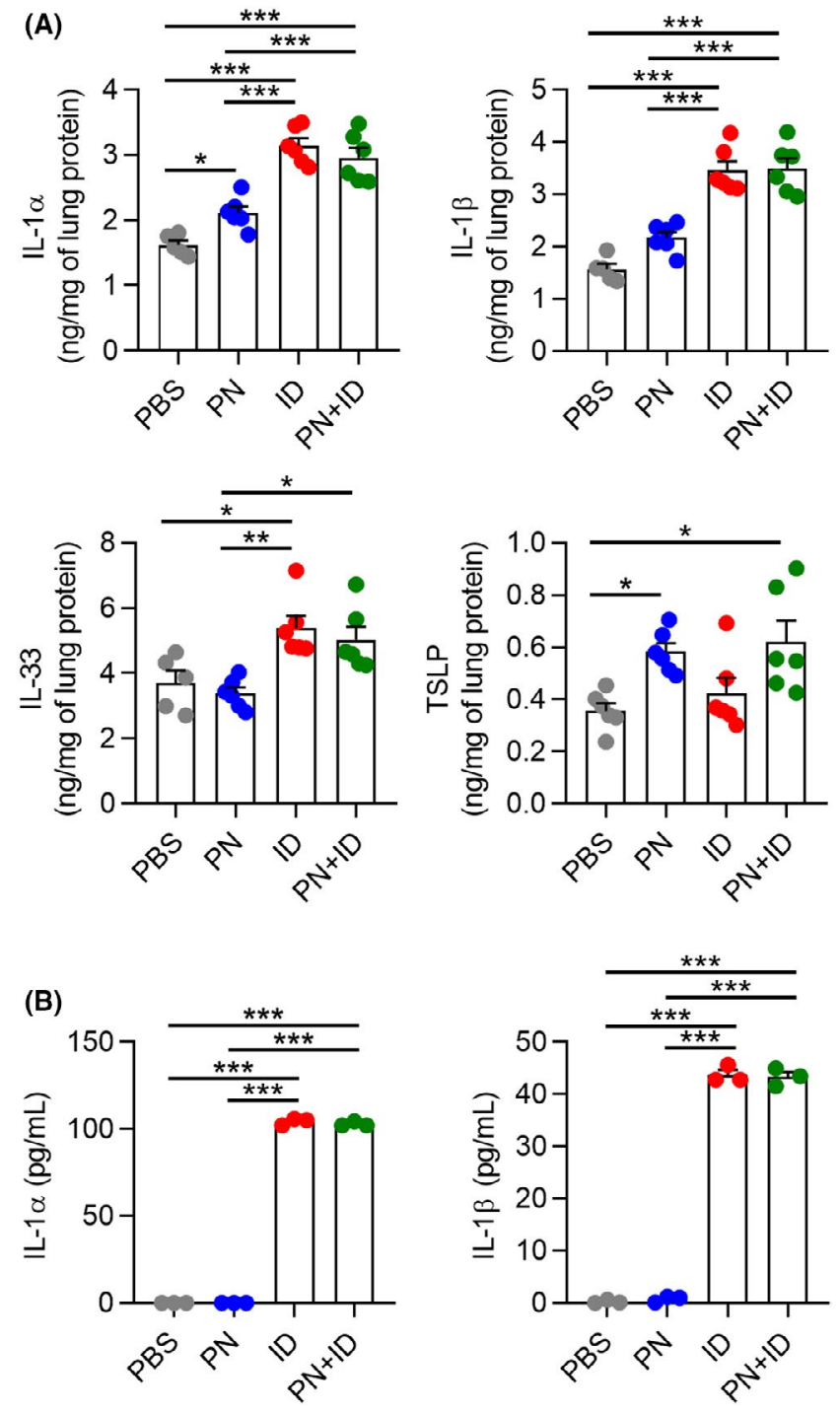

FIGURE 3 ID induces innate cytokine production in murine lungs and human bronchial epithelial cells. A, Quantities of IL-1 $\alpha$, IL-1 $\beta$, IL-33, and TSLP in mouse lung homogenates at $6 \mathrm{~h}$ following exposure to inhaled PBS, PN, ID, or PN + ID. Bars represent mean \pm SEM, and individual data points are shown $(n=5-6$ mice per group). B, Quantities of IL- $1 \alpha$ and IL-1 $\beta$ in apical cell washes from primary human bronchial epithelial cells at $4 \mathrm{~h}$ following treatment with PBS, PN, ID, or PN + ID. Bars represent mean \pm SEM, and individual data points are shown $\left(\mathrm{n}=3\right.$ donors). ${ }^{*} \mathrm{P}<.05,{ }^{* *} \mathrm{P}<.01$, ${ }^{* * *} P<.001$, one-way ANOVA. ID, indoor dust; PN, peanut

\section{4 | Indoor dust enhances activation and migration of peanut-laden $\mathrm{CDC} 1 \mathrm{~s}$ to lung-draining $\mathrm{LNs}$}

Through their ability to capture antigens and stimulate naïve T cells, cDCs play a critical role in initiating adaptive immune responses against inhaled allergens. ${ }^{21}$ Two major subsets of $\mathrm{CDC}$ s have been described in the lungs: $C D 103^{+} X C R 1^{+} \mathrm{CDC} 1 \mathrm{~s}$ and $\mathrm{CD} 11 \mathrm{~b}^{+} \mathrm{SIRP} \alpha^{+}$ $\mathrm{CDC} 2 \mathrm{~s} .{ }^{56}$ Both $\mathrm{CDC} 1 \mathrm{~s}$ and $\mathrm{CDC} 2 \mathrm{~s}$ have been reported to capture and shuttle inhaled antigens to LNs. ${ }^{33,34}$ To determine whether ID affects antigen uptake by lung cDCs, we administered fluorescent-labelled peanut allergen (PN-AF647) to the airways of mice either alone or in combination with ID. After 24 hours, lungs were harvested and uptake of PN-AF647 by lung CDC subsets was determined by flow cytometry (Figure S1A). Uptake of inhaled PN-AF647 was similar between lung $\mathrm{CDC} 1 \mathrm{~s}$ and $\mathrm{CDC} 2 \mathrm{~s}$ and was not affected by coadministration of ID, indicating that ID did not enhance antigen capture by DCs (Figure 4A,B).

In addition to antigen capture, migration of lung CDCs to $L N s$ is necessary for optimal induction of adaptive immune responses. ${ }^{57}$ To determine whether inhaled ID affected migration of DCs to lungdraining $\mathrm{mLNs}$, we administered peanut with or without ID to the airways of mice. After 24 hours, lung-draining $\mathrm{mLNs}$ were harvested and the frequencies of migratory and resident CDCs were determined by flow cytometry (Figure S1B). Compared to peanut alone, ID exposure markedly increased the number of migratory lung cDCs in $\mathrm{mLNs}$ (Figure 4C). In addition, inhaled ID resulted in activation of migratory cDCs, as indicated by increased costimulatory molecule expression (Figure 4D) compared to peanut exposure alone. To measure migration of antigen-laden DCs to $\mathrm{mLNs}$, we administered PN-AF647 with or without ID to the airways. After 24 hours, the percentage of peanut-laden ( $\mathrm{PN}-\mathrm{AF} 647^{+}$) $\mathrm{CDCs}$ in $\mathrm{mLNs}$ was determined by flow cytometry. Interestingly, nearly $30 \%$ of migratory cDC1s were $\mathrm{PN}$ $\mathrm{AF} 647^{+}$compared to about $5 \%$ of $\mathrm{cDC} 2 \mathrm{~s}$ (Figure $4 \mathrm{E}$ ). Coexposure to ID did not significantly increase the percentage of PN-AF647 $7^{+}$ cDC1s or cDC2s in mLNs (Figure 4E). However, the absolute number of peanut-laden $\mathrm{CDC} 1 \mathrm{~s}$ was dramatically increased in $\mathrm{mLNs}$ from ID-exposed mice (Figure 4F). In summary, inhaled ID induced the activation and migration of peanut-laden $\mathrm{CDC} 1 \mathrm{~s}$ to lung-draining $\mathrm{LNs}$.

\subsection{Inhaled ID induces peanut-specific Th2 cells and accumulation of Tfh cells in lung- draining LNs}

The induction of IL-4-secreting Th cells is necessary for the development of peanut-specific IgE production by B cells. Therefore, we investigated whether ID promoted IL-4 production by peanut-specific $T$ cells in lung-draining $L N s$. Mice were exposed to inhaled peanut, ID, or both twice weekly. After 2 weeks, $\mathrm{mLN}$ cells from exposed mice were collected and stimulated ex vivo with peanut allergen to assess Th cell cytokine production. Upon stimulation with peanut allergen, $\mathrm{mLN}$ cells from mice exposed to peanut and ID, but neither alone, produced the Th2 cytokines IL-4, IL-5, and IL-13 (Figure 5). Interestingly, $\mathrm{mLN}$ cells from mice exposed to peanut and ID also produced IFN- $\gamma$, suggesting that ID induced a mixed Th1/Th2 response to inhaled peanut (Figure 5). We did not observe consistent production of IL-17A by peanut-stimulated $\mathrm{mLN}$ cells, indicating that neither peanut nor ID had significant Th17 adjuvant activity (data not shown).

IL-4 is traditionally considered to be a marker of Th2 differentiation. ${ }^{25}$ However, Tfh cells are also a major source of IL-4 in LNs and are necessary for IgE responses against inhaled allergens. ${ }^{15,27,28}$ Therefore, we investigated whether ID influenced the number of Tfh cells in lung-draining $\mathrm{mLNs}$. Compared to peanut or ID alone, coexposure to inhaled peanut and ID for two weeks resulted 
(A)

DC
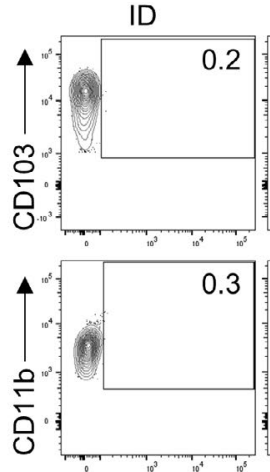

PN-AF647

(C)

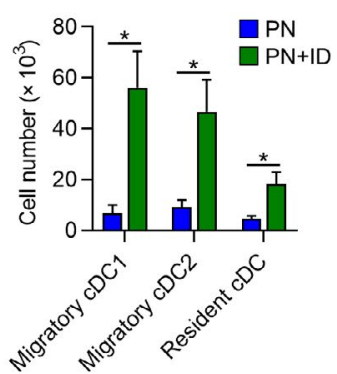

(E)

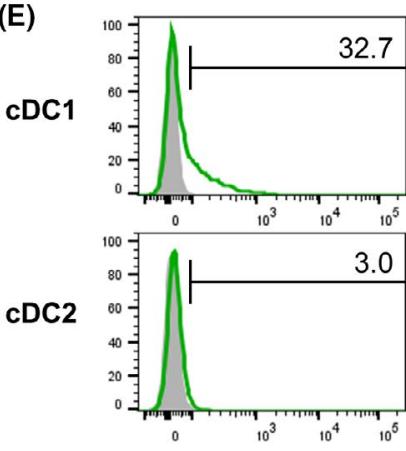

PN

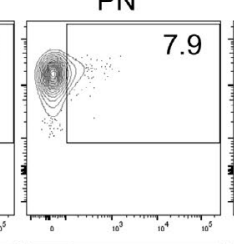

$\mathrm{PN}+\mathrm{ID}$

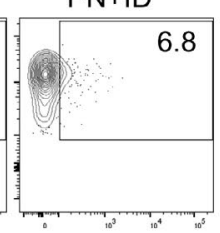

8.1

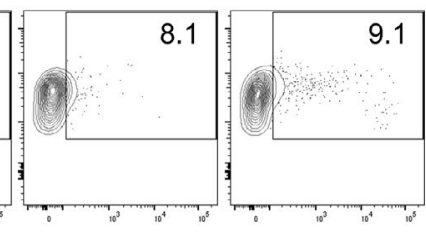

9.1

(D)
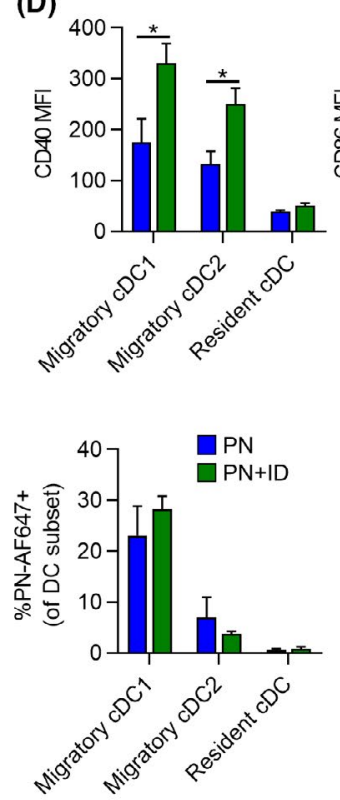

(B)
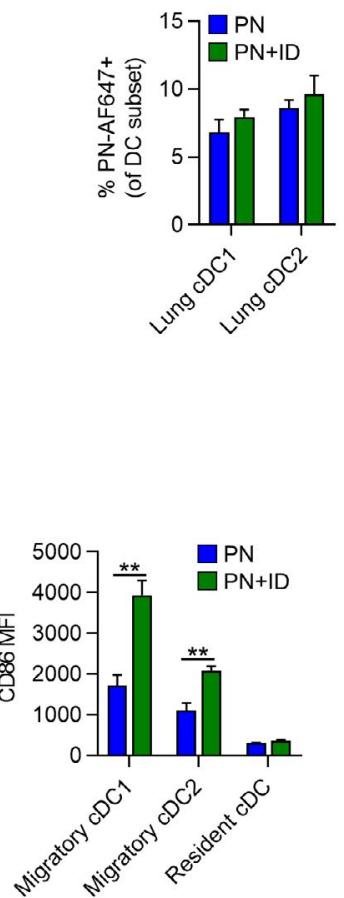

(F)



FIGURE 4 Indoor dust enhances migration of peanut-laden $\mathrm{CDC} 1 \mathrm{~s}$ to lung-draining LNs. A, B, Flow cytometric analysis of lung $C D C$ subsets $24 \mathrm{~h}$ after airway fluorescent PN-AF647 antigen alone or combined with ID. A, Representative cytograms of PN-AF647 uptake by lung $\mathrm{CDC1}$ or $\mathrm{CDC} 2$ subsets. Mice treated with ID alone were included for gating controls. Numbers represent the frequency of cells within each gate. $\mathrm{B}$, Percentage of PN-AF647 $7^{+} \mathrm{CDC1s}$ and cDC2s in lungs. C-F, Flow cytometric analysis of migratory and resident CDC subsets in lung-draining $\mathrm{mLNs}$ at $24 \mathrm{~h}$ following airway instillation of $\mathrm{PN} \pm$ ID.

$C$, Total number of migratory and resident CDC subsets in LNs. D, Median fluorescence intensity (MFI) of CD40 and $\mathrm{CD} 86$ expression by $\mathrm{CDC}$ subsets in LNs. E, Representative histograms (left) of PN-AF647 uptake by migratory $\mathrm{CDC1s}$ or CDC2s in LNs (green histograms). Mice receiving ID alone were included as gating controls (gray histograms). The percentage of $\mathrm{PN}-\mathrm{AF} 647^{+} \mathrm{CDC}$ subsets in LNs is displayed in the graph (right). $\mathrm{F}$, Total number of PN-AF $647^{+}$migratory and resident $\mathrm{CDC}$ subsets in LNs. Bars represent means \pm SEM $(n=4-5$ mice per group). Data shown are from a single experiment, representative of two experiments. ${ }^{*} P<.05,{ }^{* *} P<.01$, Student's $t$ test. ID, indoor dust; PN, peanut in a significant increase in the percentage and total number of $\mathrm{PD}^{+} \mathrm{CXCR}^{+}$Tfh cells in $\mathrm{mLNs}$ (Figure 6A,B). The increase in Tfh cells was also associated with an increase in the number of $\mathrm{CD} 19^{+} \mathrm{B}$ cells in $\mathrm{mLN}$ (Figure 6C). These findings, along with the significantly elevated peanut-specific immunoglobulin levels (Figure 1B) in mice exposed to peanut and ID, suggest that ID is a potent adjuvant for IgE responses against inhaled antigens.

\section{4 | DISCUSSION}

The increasing prevalence of PA cannot be explained by genetics alone, indicating that environmental factors play an important role in PA development. Because children spend the vast majority of their lives indoors, it is likely that exposures within the home environment influence allergic disease development. ${ }^{58}$ Within the home, children are exposed to a variety of immunostimulatory molecules, including allergens and microbial products, which can act as adjuvants in the respiratory tract and promote allergic sensitization to inhaled antigens. ${ }^{36,41,42,45}$ While indoor environmental exposures are known to contribute to wheezing and asthma inception in children, ${ }^{59}$ there is increasing evidence that the home environment may also impact food allergy development. ${ }^{9,60,61}$ Although levels of peanut allergen in the home have been positively associated with peanut sensitization, it is likely that immunogenic agents within the indoor environment also influence PA development. Using an animal model that mimics indoor exposure to environmental peanut, we found that inhalational coexposure to peanut and ID resulted in sensitization and PA development. Inhaled ID activated innate immune responses in the lungs and induced migration of peanut-laden CDCs to lung-draining LNs. In addition, inhaled ID promoted the development of peanut-specific Th2 and accumulation of Tfh cells in lung-draining LNs, which was associated with increased numbers of $B$ cells and production of peanut-specific IgE. Taken together, these findings suggest that in addition to the levels of peanut allergen in the home, the adjuvant activity of indoor dust is also a determinant of peanut sensitization and possibly PA development. 

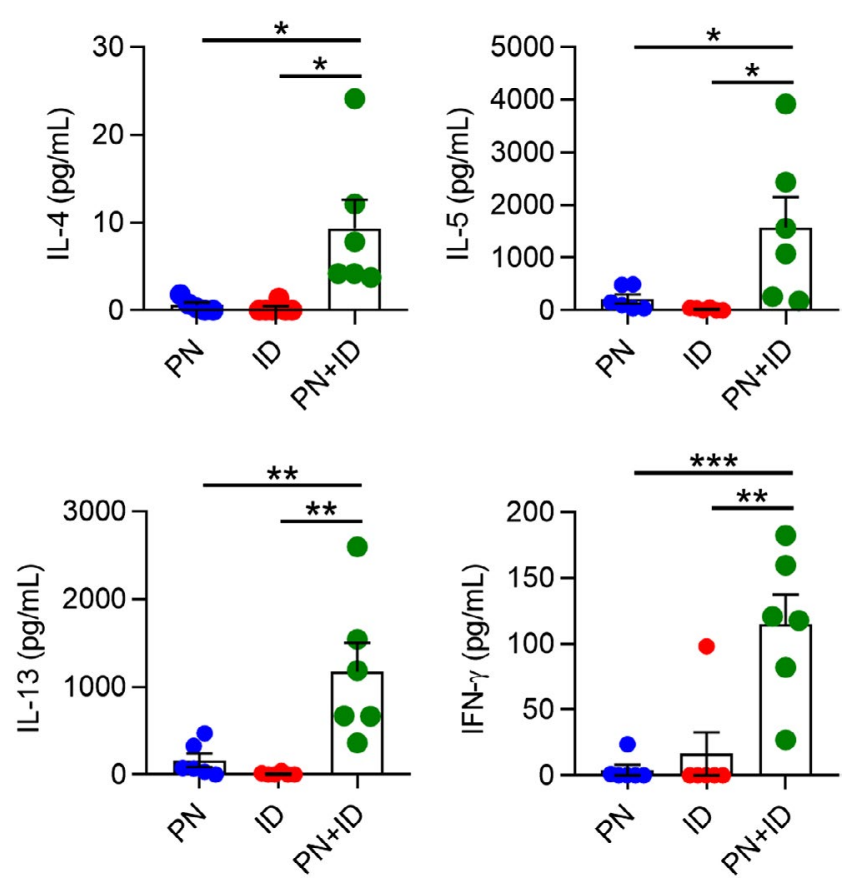

FIGURE 5 Inhaled ID promotes peanut-specific Th2 responses in lung-draining LNs. Lung-draining LN cells were collected from mice sensitized to PN, ID or PN + ID twice weekly for $2 \mathrm{wk}$, and then stimulated ex vivo with peanut antigen. Four days later, levels of IL-4, IL-5, IL-13, and IFN- $\gamma$ in cell culture supernatants were measured by ELISA. Bars represent means \pm SEM, and individual data points are shown ( $n=5-6$ mice per group). Data shown are from a single experiment, representative of two experiments. ${ }^{*} P<.05,{ }^{* *} P<.01,{ }^{* * *} P<.001$, one-way ANOVA. ID, indoor dust; PN, peanut

The classic paradigm of food allergy pathogenesis is that food allergen sensitization initially occurs in the gastrointestinal tract after oral exposure. ${ }^{62}$ However, the vast majority of children with PA react upon their first known ingestion of peanut, ${ }^{4}$ suggesting that sensitization may have occurred through a non-enteral route. Furthermore, the landmark Learning Early About Peanut Allergy (LEAP) trial demonstrated that oral exposure to peanut during infancy resulted in tolerance instead of allergy. ${ }^{5}$ To account for these observations, the dual-allergen exposure hypothesis was recently proposed as a model for food allergy development during early childhood. ${ }^{63}$ This hypothesis postulates that early oral exposure to food allergens induces tolerance, whereas exposure at non-enteral sites, such as the skin or respiratory tract, results in food sensitization and allergy development. In support of the dual-allergen hypothesis, animal studies have shown that cutaneous or inhalational exposure to food allergens results in sensitization. ${ }^{15-17,64,65}$ In humans, application of peanut oil to eczematous skin has been associated with PA development. ${ }^{10}$ In addition, high levels of environmental peanut exposure (as estimated by household peanut consumption) are associated with increased risk for PA; however, this risk was mitigated by early oral exposure to peanut. ${ }^{7}$ Moreover, peanut allergen levels in household dust directly correlate with rates of peanut sensitization and probable allergy in children. ${ }^{8,9}$ The effect of environmental peanut exposure on PA risk is augmented in children with severe atopic dermatitis ${ }^{9}$ or filaggrin loss-of-function mutations, ${ }^{8}$ suggesting that an impaired skin barrier facilitates epicutaneous sensitization to peanut in household dust. While most human studies have focused on cutaneous exposure to environmental peanut, sensitization may also occur through the respiratory tract. Although peanut allergen is not thought to be airborne under ambient conditions, ${ }^{11}$ disruption of dust during domestic activity could conceivably lead to airborne peanut allergens, as has been observed for house dust mite allergens. ${ }^{66}$ Additionally, peanut-specific $\mathrm{CD} 4^{+} \mathrm{T}$ cells from PA subjects have increased expression of the airway-homing chemokine receptor CCR4 but not the skin-homing molecule cutaneous lymphocyte-associated antigen (CLA) or skin-homing chemokine receptor CCR10, suggesting that peanut sensitization occurred in the lungs. ${ }^{18}$ Thus, both the skin and respiratory tract are plausible sites for sensitization to environmental peanut.

Indoor dust contains an environmentally relevant mixture of immunomodulatory agents to which children are exposed. ${ }^{67}$ Exposure to several of these agents, including indoor allergens and endotoxin, has been associated with an increased risk of asthma development. ${ }^{68,69}$ However, many observational studies suggest that earlylife exposure to allergens and microbes confers protection against asthma. $^{70-73}$ The reason for this apparent discrepancy is unclear, but may depend upon the composition of the home microbiota or other environmental factors such as tobacco smoke exposure. ${ }^{74}$ In contrast to asthma, relatively little is known regarding how indoor environmental factors impact food allergy development. In a cohort from the Urban Environment and Childhood Asthma study, neither food sensitization nor food allergy were associated with exposure to indoor allergens during the first year of life. ${ }^{60}$ Interestingly, higher endotoxin levels were associated with protection against the development of overall food allergy, but not peanut allergy. ${ }^{60}$ The indoor dust sample used for our studies had low levels of endotoxin, which may facilitate allergic airway sensitization to inhaled antigens. ${ }^{75}$ It will be interesting to determine whether indoor dust from homes with higher levels of endotoxin or other microbial-derived products prevents sensitization to inhaled peanut. It is also possible that the presence of certain immunogenic molecules in indoor dust is a prerequisite for airway sensitization to inhaled peanut. While the identity of these components is unknown, we have found that heattreated ID retains its adjuvant activity (data not shown), suggesting that proteases or other heat-sensitive proteins are not involved. Identifying the key components of indoor dust that promote allergic airway sensitization is currently underway.

During allergic sensitization, allergens or environmental adjuvants trigger airway epithelial cells to release innate cytokines (IL-1, IL-33, TSLP) that promote the development of Th2 responses. ${ }^{20} \mathrm{We}$ observed that acute exposure to inhaled ID triggered the release of innate cytokines in murine lungs, which was associated with the development of peanut-specific Th2 cells in lung-draining LNs. Although Th2 cells have historically been considered necessary for IgE responses against inhaled allergens, there is growing evidence that Tfh cells are also important. ${ }^{15,27}$ Indeed, a recent report by 
(A)

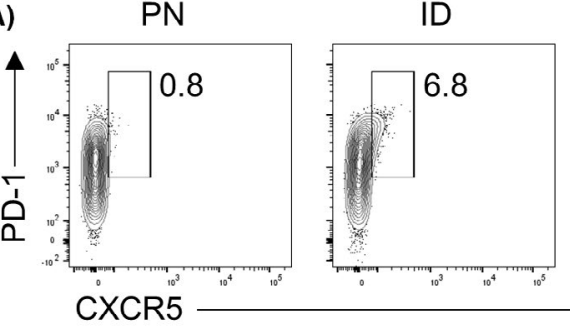

$\mathrm{PN}+\mathrm{ID}$

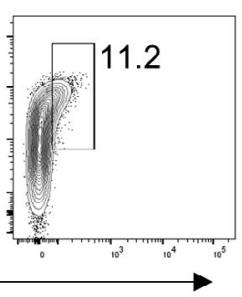

(B)





(C)

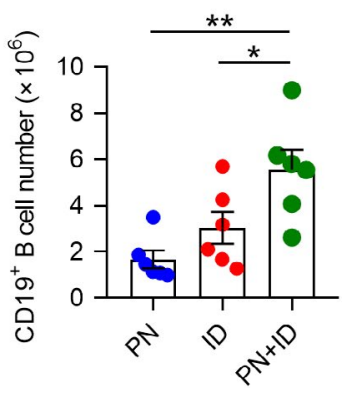

FIGURE 6 Inhaled ID results in increased T follicular helper cells in lung-draining LNs. A-C, Analysis of Tfh cells in lung-draining LNs from mice after two weeks of airway sensitization with either PN, ID, or PN + ID. A, Representative cytograms showing the frequency of PD $-1^{+} \mathrm{CXCR} 5^{+}$Tfh after gating on $\mathrm{CD} 3^{+} \mathrm{CD} 4^{+} \mathrm{CD} 44^{\text {hi }}$ cells in $\mathrm{mLN}$. B, Percentage (left) and total number (right) of $\mathrm{CD} 3^{+} \mathrm{CD} 4^{+} \mathrm{CD} 44^{\text {hi }} \mathrm{PD}-1^{+} \mathrm{CXCR} 5^{+}$Tfh cells in lung-draining LNs. C, Number of CD19 ${ }^{+} \mathrm{B}$ cells in lung-draining LNs at two weeks following airway sensitization with either PN, ID, or PN + ID. Bars represent means $\pm S E M$, and individual data points are shown ( $n=6$ mice per group). Data shown are from a single experiment, representative of two experiments. ${ }^{*} P<.05,{ }^{* *} P<.01,{ }^{* * *} P<.001$, one-way ANOVA. ID, indoor dust; PN, peanut

Dolence et al found that Tfh cells were essential for IgE responses against inhaled peanut. ${ }^{15}$ In that study, inhaled peanut flour induced production of IL- $1 \alpha$ and IL-1 $\beta$, which were critical for the development of peanut-specific Tfh cells. We found that peanut extract induced only low levels of IL-1 in the lungs, which is likely due to weaker endogenous adjuvant activity of peanut extract compared to peanut flour. ${ }^{15}$ In contrast, we found that inhaled ID triggered robust production of IL- $1 \alpha$, IL-1 $\beta$, and IL-33, which was associated with increased numbers of Tfh cells in the draining LNs. We also observed that ID, but not peanut extract alone, induced IL-1 $\alpha$ and IL-1 $\beta$ production by primary HBECs. While we could not detect production of TSLP or IL-33 by peanut or ID-treated HBECs, we cannot exclude the possibility that these cytokines are induced at later time-points. Indeed, peanut extract has been reported to stimulate IL-33 mRNA expression by human keratinocytes, ${ }^{64}$ suggesting that peanut allergen can directly stimulate innate responses in epithelial cells. Taken together, our findings suggest that environmental adjuvants in indoor dust can stimulate innate signalling pathways important for Tfh development and IgE production against inhaled antigens.

Through their ability to capture antigens and stimulate naïve $T$ cells, cDCs play a critical role in initiating adaptive immune responses against inhaled allergens. ${ }^{21}$ While intestinal $\mathrm{CD}^{10} 3^{+} \mathrm{cDC} 1 \mathrm{~s}$ have been reported to transport ingested peanut antigen to gut-draining $\mathrm{LNs},{ }^{76}$ the lung DC subset responsible for capturing inhaled peanut antigen and shuttling it to LNs is unknown. We found that both lung $\mathrm{CD}_{103}{ }^{+} \mathrm{cDC} 1 \mathrm{~s}$ and $\mathrm{CD} 11 \mathrm{~b}^{+} \mathrm{cDC} 2 \mathrm{~s}$ were able to take up peanut allergen from the airways. Although ID exposure did not affect antigen uptake by lung $C D C s$, it did induce activation and migration of $c D C s$ to lung-draining LN. In contrast to reports showing that $\mathrm{cDC} 1 \mathrm{~s}$ and $\mathrm{cDC} 2 \mathrm{~s}$ were equivalent in transporting inhaled antigen to $\mathrm{mLNs},{ }^{32}$ we found a greater number of peanut-laden cDC1s compared to $\mathrm{cDC} 2 \mathrm{~s}$ in $\mathrm{mLNs}$. Migration of peanut-laden $\mathrm{CDC} 1 \mathrm{~s}$ was associated with the differentiation of peanut-specific Th2 cells and increased numbers of Tfh cells in $\mathrm{LNs}$, suggesting that $\mathrm{CDC} 1 \mathrm{~s}$ may promote IgE responses against inhaled peanut. This observation is intriguing, as lung $\mathrm{CDC} 2 \mathrm{~s}$ have been reported to be the primary $\mathrm{DC}$ subset that induces allergic responses against inhaled allergens. ${ }^{33,35}$ Moreover, cDC2s were recently reported to induce Tfh cell responses against inhaled antigens, although this study was performed in the absence of Th2 adjuvants and therefore IgE responses could not be assessed. ${ }^{32}$ However, we and others have previously found that lung cDC1s from ID-exposed mice are capable of stimulating Th2 cell differentiation. ${ }^{34,42}$ Furthermore, both gut and splenic cDC1s have been shown to prime antigen-specific Tfh cells' cell responses, ${ }^{76,77}$ raising the possibility that lung $\mathrm{CDC} 1 \mathrm{~s}$ may do the same under certain conditions. It is also possible that enhanced degradation of AF647labelled peanut is responsible for the low number of peanut-laden $\mathrm{cDC} 2 \mathrm{~s}$ present in mediastinal LN. However, AF647 $7^{+} \mathrm{CDC} 2 \mathrm{~s}$ were readily detectable in $\mathrm{mLN}$ after airway delivery of other AF647-labelled allergens, suggesting that antigen processing is not inherently enhanced in this CDC subset. ${ }^{33,34}$ Identifying the lung CDC subset that promotes IgE responses against peanut allergen is under current investigation.

In summary, we have found that inhalational exposure to peanut and indoor dust results in allergic sensitization and the development of PA in a mouse model. These findings suggest that in addition to 
peanut levels within the home, the composition of environmental adjuvants in household dust is also an important determinant for PA development in children. Identifying the key components of indoor dust that either promote or protect against peanut sensitization may lead to novel environmental interventions aimed at reducing PA development in at-risk children.

\section{ACKNOWLEDGEMENTS}

We thank Wesley Burks, Claire Doerschuk, and Stephen Tilley for the generous provision of laboratory equipment and other scientific resources; Donald Cook, Hideki Nakano, David Lauzier, and Kelly Orgel for assistance with data interpretation and technical assistance; the Center for Environmental Medicine, Asthma and Lung Biology for flow cytometry services; and the Tissue Procurement and Cell Culture Core of the Marsico Lung Institute and John Minges for provision and assistance with human bronchial epithelial cell cultures.

\section{CONFLICT OF INTEREST}

The authors have no conflicts of interest to disclose.

\section{DATA AVAILABILITY STATEMENT}

The data that support the findings of this study are available from the corresponding author upon reasonable request.

\section{ORCID}

Johanna M. Smeekens (iD https://orcid.org/0000-0001-9651-3852

Michael D. Kulis iD https://orcid.org/0000-0001-8092-7444

\section{REFERENCES}

1. Sicherer SH, Sampson HA. Food allergy: Epidemiology, pathogenesis, diagnosis, and treatment. J Allergy Clin Immunol. 2014;133(2):291-307; quiz 308.

2. Sicherer SH, Munoz-Furlong A, Godbold JH, Sampson HA. US prevalence of self-reported peanut, tree nut, and sesame allergy: 11year follow-up. J Allergy Clin Immunol. 2010;125(6):1322-1326.

3. Skolnick HS, Conover-Walker MK, Koerner CB, Sampson HA, Burks W, Wood RA. The natural history of peanut allergy. J Allergy Clin Immunol. 2001;107(2):367-374.

4. Bock SA, Munoz-Furlong A, Sampson HA. Fatalities due to anaphylactic reactions to foods. J Allergy Clin Immunol. 2001;107(1):191-193.

5. Du Toit G, Roberts G, Sayre PH, et al. Randomized trial of peanut consumption in infants at risk for peanut allergy. $N$ Engl $J$ Med. 2015;372(9):803-813.

6. Osborne NJ, Koplin JJ, Martin PE, et al. Prevalence of challengeproven IgE-mediated food allergy using population-based sampling and predetermined challenge criteria in infants. J Allergy Clin Immunol. 2011;127(3):668-676.e661-662.

7. Fox AT, Sasieni P, du Toit G, Syed H, Lack G. Household peanut consumption as a risk factor for the development of peanut allergy. $J$ Allergy Clin Immunol. 2009;123(2):417-423.
8. Brough HA, Simpson A, Makinson K, et al. Peanut allergy: effect of environmental peanut exposure in children with filaggrin lossof-function mutations. J Allergy Clin Immunol. 2014;134(4):867-875 e861.

9. Brough HA, Liu AH, Sicherer S, et al. Atopic dermatitis increases the effect of exposure to peanut antigen in dust on peanut sensitization and likely peanut allergy. J Allergy Clin Immunol. 2015;135(1):164-170.

10. Lack G, Fox D, Northstone K, Golding J. Factors associated with the development of peanut allergy in childhood. N Engl J Med. 2003;348(11):977-985.

11. Brough HA, Makinson K, Penagos M, et al. Distribution of peanut protein in the home environment. J Allergy Clin Immunol. 2013;132(3):623-629.

12. Brough HA, Santos AF, Makinson K, et al. Peanut protein in household dust is related to household peanut consumption and is biologically active. J Allergy Clin Immunol. 2013;132(3):630-638.

13. Brough HA, Kull I, Richards K, et al. Environmental peanut exposure increases the risk of peanut sensitization in high-risk children. Clin Exp Allergy. 2018;48(5):586-593.

14. Sicherer SH, Burks AW, Sampson HA. Clinical features of acute allergic reactions to peanut and tree nuts in children. Pediatrics. 1998;102(1):e6.

15. Dolence JJ, Kobayashi T, lijima K, et al. Airway exposure initiates peanut allergy by involving the IL-1 pathway and T follicular helper cells in mice. J Allergy Clin Immunol. 2018;142(4):1144-1158 e1148.

16. Dunkin D, Berin MC, Mayer L. Allergic sensitization can be induced via multiple physiologic routes in an adjuvant-dependent manner. $J$ Allergy Clin Immunol. 2011;128(6):1251-1258.e1252.

17. Wavrin S, Bernard H, Wal JM, Adel-Patient K. Cutaneous or respiratory exposures to peanut allergens in mice and their impacts on subsequent oral exposure. Int Arch Allergy Immunol. 2014;164(3):189-199.

18. Blom LH, Juel-Berg N, Larsen LF, Hansen KS, Poulsen LK. Circulating allergen-specific TH2 lymphocytes: CCR18(+) rather than CLA(+) is the predominant phenotype in peanut-allergic subjects. J Allergy Clin Immunol. 2018;141(4):1498-1501.e1495.

19. Roberts JW, Wallace LA, Camann DE, et al. Monitoring and reducing exposure of infants to pollutants in house dust. Rev Environ Contam Toxicol. 2009;201:1-39.

20. Lambrecht BN, Hammad H. Allergens and the airway epithelium response: gateway to allergic sensitization. J Allergy Clin Immunol. 2014;134(3):499-507.

21. Lambrecht $\mathrm{BN}, \mathrm{Hammad} \mathrm{H}$. Lung dendritic cells in respiratory viral infection and asthma: from protection to immunopathology. Ann Rev Immunol. 2012;30:243-270.

22. Willart MA, Deswarte K, Pouliot P, et al. Interleukin-1alpha controls allergic sensitization to inhaled house dust mite via the epithelial release of GM-CSF and IL-33. J Exp Med. 2012;209(8):1505-1517.

23. Chu DK, Llop-Guevara A, Walker TD, et al. IL-33, but not thymic stromal lymphopoietin or IL-25, is central to mite and peanut allergic sensitization. J Allergy Clin Immunol. 2013;131(1):187-200 e181-188.

24. Zhou B, Comeau MR, De Smedt T, et al. Thymic stromal lymphopoietin as a key initiator of allergic airway inflammation in mice. Nat Immunol. 2005;6(10):1047-1053.

25. Paul WE, Zhu J. How are $T(H) 2$-type immune responses initiated and amplified? Nat Rev Immunol. 2010;10(4):225-235.

26. Meli AP, Fontes G, Leung Soo C, King I. T follicular helper cell-derived IL-4 is required for IgE production during intestinal helminth infection. J Immunol. 2017;199(1):244-252.

27. Kobayashi T, lijima K, Dent AL, Kita H. Follicular helper T cells mediate IgE antibody response to airborne allergens. J Allergy Clin Immunol. 2017;139(1):300-313 e307. 
28. Noble A, Zhao J. Follicular helper T cells are responsible for IgE responses to Der $\mathrm{p} 1$ following house dust mite sensitization in mice. Clin Exp Allergy. 2016;46(8):1075-1082.

29. Vinuesa CG, Linterman MA, Yu D, MacLennan IC. Follicular Helper T Cells. Ann Rev Immunol. 2016;34:335-368.

30. Choi YS, Kageyama R, Eto D, et al. ICOS receptor instructs T follicular helper cell versus effector cell differentiation via induction of the transcriptional repressor Bcl6. Immunity. 2011;34(6):932-946.

31. Goenka R, Barnett LG, Silver JS, et al. Cutting edge: dendritic cellrestricted antigen presentation initiates the follicular helper $\mathrm{T}$ cell program but cannot complete ultimate effector differentiation. J Immunol. 2011;187(3):1091-1095.

32. Krishnaswamy JK, Gowthaman U, Zhang B, et al. Migratory $\mathrm{CD} 11 \mathrm{~b}(+)$ conventional dendritic cells induce $\mathrm{T}$ follicular helper celldependent antibody responses. Sci Immunol. 2017;2(18): eaam9169.

33. Plantinga M, Guilliams M, Vanheerswynghels M, et al. Conventional and monocyte-derived $\mathrm{CD} 11 \mathrm{~b}(+)$ dendritic cells initiate and maintain T helper 2 cell-mediated immunity to house dust mite allergen. Immunity. 2013;38(2):322-335.

34. Nakano H, Free ME, Whitehead GS, et al. Pulmonary CD103(+) dendritic cells prime Th2 responses to inhaled allergens. Mucosal Immunol. 2012;5(1):53-65.

35. Zhou Q, Ho AW, Schlitzer A, et al. GM-CSF-licensed CD11b+ lung dendritic cells orchestrate Th2 immunity to Blomia tropicalis. J Immunol. 2014;193(2):496-509.

36. Boasen J, Chisholm D, Lebet L, Akira S, Horner AA. House dust extracts elicit Toll-like receptor-dependent dendritic cell responses. J Allergy Clin Immunol. 2005;116(1):185-191.

37. Kim H, Tse K, Levin L, et al. House dust bioactivities predict skin prick test reactivity for children with high risk of allergy. J Allergy Clin Immunol. 2012;129(6):1529-1537.e1522.

38. Van Dyken SJ, Garcia D, Porter P, et al. Fungal Chitin from asthmaassociated home environments induces eosinophilic lung infiltration. J Immunol. 2011;187(5):2261-2267.

39. Porter P, Susarla SC, Polikepahad S, et al. Link between allergic asthma and airway mucosal infection suggested by proteinase-secreting household fungi. Mucosal Immunol. 2009;2(6):504-517.

40. Ng N, Lam D, Paulus P, Batzer G, Horner AA. House dust extracts have both TH2 adjuvant and tolerogenic activities. J Allergy Clin Immunol. 2006;117(5):1074-1081.

41. Wilson RH, Maruoka S, Whitehead GS, et al. The Toll-like receptor 5 ligand flagellin promotes asthma by priming allergic responses to indoor allergens. Nat Med. 2012;18(11):1705-1710.

42. Moran TP, Nakano K, Whitehead GS, Thomas SY, Cook DN, Nakano $\mathrm{H}$. Inhaled house dust programs pulmonary dendritic cells to promote type $2 \mathrm{~T}$-cell responses by an indirect mechanism. Am J Physiol Lung Cell Mol Physiol. 2015;309(10):L1208-1218.

43. Shalaby KH, Lyons-Cohen MR, Whitehead GS, et al. Pathogenic $\mathrm{TH} 17$ inflammation is sustained in the lungs by conventional dendritic cells and Toll-like receptor 4 signaling. J Allergy Clin Immunol. 2017;142(4):1229-1242.

44. Gough L, Sewell HF, Shakib F. The proteolytic activity of the major dust mite allergen Der $\mathrm{p} 1$ enhances the IgE antibody response to a bystander antigen. Clin Exp Allergy. 2001;31(10):1594-1598.

45. Whitehead GS, Thomas SY, Shalaby KH, et al. TNF is required for TLR ligand-mediated but not protease-mediated allergic airway inflammation. J Clin Invest. 2017;127(9):3313-3326.

46. Bednar KJ, Hardy L, Smeekens J, et al. Antigenic liposomes for generation of disease-specific antibodies. J Vis Exp. 2018;(140): e58285.

47. Sever ML, Arbes SJ Jr, Gore JC, et al. Cockroach allergen reduction by cockroach control alone in low-income urban homes: a randomized control trial. J Allergy Clin Immunol. 2007;120(4):849-855.

48. Wilson RH, Whitehead GS, Nakano H, Free ME, Kolls JK, Cook DN. Allergic sensitization through the airway primes Th17-dependent neutrophilia and airway hyperresponsiveness. Am J Respir Crit Care Med. 2009;180(8):720-730.

49. Orgel K, Smeekens JM, Ye P, et al. Genetic diversity between mouse strains allows identification of the CCO27/GeniUnc strain as an orally reactive model of peanut allergy. J Allergy Clin Immunol. 2018;143(3):1027-1037.

50. Fulcher ML, Randell SH. Human nasal and tracheo-bronchial respiratory epithelial cell culture. Methods Mol Biol. 2013;945:109-121.

51. Moran TP, Nakano H, Kondilis-Mangum HD, Wade PA, Cook DN. Epigenetic control of Ccr7 expression in distinct lineages of lung dendritic cells. J Immunol. 2014;193(10):4904-4913.

52. Nakano $\mathrm{H}$, Cook DN. Pulmonary antigen presenting cells: isolation, purification, and culture. Methods Mol Biol. 2013;1032:19-29.

53. Sheehan WJ, Brough HA, Makinson K, Petty CR, Lack G, Phipatanakul W. Distribution of peanut protein in school and home environments of inner-city children. J Allergy Clin Immunol. 2017;140(6):1724-1726.

54. U.S. EPA. Exposure Factors Handbook 2011 Edition (Final Report). U.S. Environmental Protection Agency, Washington, DC, EPA/600/ R-09/052F, 2011. In: 2011.

55. Pulendran B, Artis D. New paradigms in type 2 immunity. Science. 2012;337(6093):431-435.

56. Guilliams M, Dutertre CA, Scott CL, et al. Unsupervised high-dimensional analysis aligns dendritic cells across tissues and species. Immunity. 2016;45(3):669-684.

57. Worbs T, Hammerschmidt SI, Forster R. Dendritic cell migration in health and disease. Nat Rev Immunol. 2017;17(1):30-48.

58. Platts-Mills TA. The allergy epidemics: 1870-2010. J Allergy Clin Immunol. 2015;136(1):3-13.

59. Ahluwalia SK, Matsui EC. The indoor environment and its effects on childhood asthma. Curr Opin Allergy Clin Immunol. 2011;11(2):137-143.

60. McGowan EC, Bloomberg GR, Gergen PJ, et al. Influence of earlylife exposures on food sensitization and food allergy in an inner-city birth cohort. J Allergy Clin Immunol. 2015;135(1):171-178.

61. Garcia-Boyano M, Pedrosa M, Quirce S, Boyano-Martinez T. Household almond and peanut consumption is related to the development of sensitization in young children. J Allergy Clin Immunol. 2016;137(4):1248-1251.e1246.

62. Burks AW. Peanut allergy. Lancet. 2008;371(9623):1538-1546.

63. Turcanu V, Brough HA, Du Toit G, et al. Immune mechanisms of food allergy and its prevention by early intervention. Curr Opin Immunol. 2017;48:92-98.

64. Tordesillas L, Goswami R, Benede S, et al. Skin exposure promotes a Th2-dependent sensitization to peanut allergens. J Clin Invest. 2014;124(11):4965-4975.

65. Noti M, Kim BS, Siracusa MC, et al. Exposure to food allergens through inflamed skin promotes intestinal food allergy through the thymic stromal lymphopoietin-basophil axis. J Allergy Clin Immunol. 2014;133(5):1390-1399, 1399.e1391-1396.

66. Tovey ER, Chapman MD, Wells CW, Platts-Mills TA. The distribution of dust mite allergen in the houses of patients with asthma. Am Rev Respir Dis. 1981;124(5):630-635.

67. Batzer G, Lam DP, Paulus P, Boasen J, Ng N, Horner AA. Using house dust extracts to understand the immunostimulatory activities of living environments. Immunobiology. 2007;212(6):491-498.

68. Litonjua AA, Carey VJ, Burge HA, Weiss ST, Gold DR. Exposure to cockroach allergen in the home is associated with incident doctordiagnosed asthma and recurrent wheezing. J Allergy Clin Immunol. 2001;107(1):41-47.

69. Thorne PS, Kulhankova K, Yin M, Cohn R, Arbes SJ Jr, Zeldin DC. Endotoxin exposure is a risk factor for asthma: the national survey of endotoxin in United States housing. Am J Respir Crit Care Med. 2005;172(11):1371-1377. 
70. Lynch SV, Wood RA, Boushey H, et al. Effects of early-life exposure to allergens and bacteria on recurrent wheeze and atopy in urban children. J Allergy Clin Immunol. 2014;134(3):593-601.e512.

71. Stein MM, Hrusch CL, Gozdz J, et al. Innate immunity and asthma risk in amish and hutterite farm children. $N$ Engl J Med. 2016;375(5):411-421.

72. von Mutius E, Vercelli D. Farm living: effects on childhood asthma and allergy. Nat Rev Immunol. 2010;10(12):861-868.

73. Braun-Fahrlander C, Riedler J, Herz U, et al. Environmental exposure to endotoxin and its relation to asthma in school-age children. N Engl J Med. 2002;347(12):869-877.

74. O'Connor GT, Lynch SV, Bloomberg GR, et al. Early-life home environment and risk of asthma among inner-city children. J Allergy Clin Immunol. 2018;141(4):1468-1475.

75. Whitehead GS, Thomas SY, Cook DN. Modulation of distinct asthmatic phenotypes in mice by dose-dependent inhalation of microbial products. Environ Health Perspect. 2014;122(1):34-42.

76. Chu DK, Jimenez-Saiz R, Verschoor CP, et al. Indigenous enteric eosinophils control DCs to initiate a primary Th2 immune response in vivo. J Exper Med. 2014;211(8):1657-1672.
77. Lahoud $\mathrm{MH}$, Ahmet F, Kitsoulis S, et al. Targeting antigen to mouse dendritic cells via Clec9A induces potent CD4 T cell responses biased toward a follicular helper phenotype. J Immunol. 2011;187(2):842-850.

\section{SUPPORTING INFORMATION}

Additional supporting information may be found online in the Supporting Information section at the end of the article.

How to cite this article: Smeekens JM, Immormino RM, Balogh PA, Randell SH, Kulis MD, Moran TP. Indoor dust acts as an adjuvant to promote sensitization to peanut through the airway. Clin Exp Allergy. 2019;49:1500-1511. https://doi. org/10.1111/cea.13486 